\title{
A Review on Stool Transplantation
}

\section{Rajani Chowdary A*}

Department of Microbiology and Food science and Technology Gitam Institute of Science, Gitam University, India

*Corresponding author: Rajani Chowdary A, Department of Microbiology and Food science and Technology Gitam Institute of Science, Gitam University, India, Tel: +91-8985945210; Email: rajaniagis@gmail.com

\section{Mini Review}

Volume 2 Issue 1

Received Date: March 15, 2018

Published Date: March 26, 2018

\section{Abstract}

The gut microbiota is composed of a significant number of different bacteria, approximately 160 species per person per fecal sample. Its quality depends upon the individual eating habits usually dictated by its social, economical and environmental circumstances. There has been increasing attention in understanding the role of the human gut flora to reveal the therapeutic prospective of its manipulation. Now-a-days clinicians will treat the bacterial infections specifically Clostridium difficile with a process called fecal matter transplantation (FMT), which involves extracting healthy bacteria from another individual's fecal matter and then processing and transferring that bacteria to the infected patient directly. This process usually done with colonoscopy, endoscopy or enema, and usually does not permanently resolves the issue, leading to ongoing FMTs over time. There are preliminary indications to put forward that it may also carry therapeutic potential for other diseases like inflammatory bowel disease, obesity, metabolic syndrome, and functional gastrointestinal disorders.

Keywords: Stool transplantation; Gut microbiota; Clostridium difficile infection; Colonoscopy.

\section{Introduction}

Stool transplants also known as fecal microbiota transplant or fecal transfusion or fecal enema or fecal bacteriopathy or human probiotic infusion. In this procedure the healthy human complex fecal microbiota is transplanted into ill patient for the restoration of colonic bacteria which are suppressed or killed by oral antibiotics to treat gastro-intestinal disorders [1]. Transplantation can be done by various modalities like enema, oro-gastric tube or colonoscopy or through naso-gastric intubation or directly into the mouth in the form of capsules containing freeze dried stool material. The human gastrointestinal microbiota is dense and contains diversed variety of bacterial flora which colonize the human body specifically colon as a common habitat. They play an important role in the colonization resistance or act as competitors to prevent the overgrowth of pathogenic microorganisms in colon [2,3]. The usage of antibiotics may leads to replacement of normal gut microbiota with pathogens like Clostridium difficile which undergoes multiplication rapidly and dominates the gut ecosystem and cause C.difficile colitis. This further leads to debilitating condition like fatal diarrhea. It is also used to treat other gastrointestinal disorders like colitis, constipation, irritable bowel syndrome and in some neurological 


\section{Journal of Infectious Diseases \& Travel Medicine}

conditions like parkinson's disease or multiple sclerosis. FMT (Fecal microbiota transplantation) forms the first line of defense or treatment against C.difficile infection. In some countries of world it is customary for a newborn infant to feed with a small amount of mother's stool such that the baby's immune system get activated and their colon is also enriched with complex healthy human bacterial flora. It is sporadically used US individuals since from 1950's. After FDA approval the clinicians practicing this procedure to treat recurrent C.difficile infection and the treatment is more effective than vancomycin antimicrobial therapy [4]. The method is inexpensive, low risk and highly effective. Individuals with good health and not taking any antibiotics for at least six months are considered as donors of stool samples for FMT procedure. The stool samples should be screened for pathogenic microbial examination, serological tests for detection of hepatitis viruses, other enteroviruses etc and also should be examined for the presence of parasites ova and cyst. Formerly it is considered as a last resort therapy by some clinicians because of its remarkable scenery and invasiveness in contrast to antibiotics, apparent potential risk of infection transmission, and lack of Medicare coverage for donor stool, position statements by specialists in infectious diseases and other societies have been moving toward acceptance of FMT as standard therapy for relapsing C.difficile infection [5-7].

\section{Procedure of Stool Transplantation}

Before starting the procedure, huge cautions should be taken for selection and screening of the donor. Generally individuals who are test positive for certain diseases like C.difficile infection, irritable bowel syndrome, ulcerative colitis, intestinal Behcet's disease as well as any donor carrying any pathogenic gastrointestinal infectious agents like E.coli, Shigella species, Salmonella species, Vibrio cholera, Campylobacter species, Clostridium species etc and intestinal parasites are excluded as donors for transplantation. Donors must be tested for a wide array of bacterial and parasitic infections using various microbiological test procedures. Around 200 to 300 grams (7.1 to 10.6 ounces) of stool material is suggested per treatment for optimum results. Fresh stools have been recommended to be used within six hours; however frozen stool samples can also be used without loss of efficacy. The procedure includes single or multiple infusions of bacterial flora originating from a healthy donor by enema, through a colonoscope, through a nasogastric or nasoduodenal tube, or by mouth via a capsule. Fecal transplantation by colonoscopy has a good outcome and the success rate is $90 \%$ compared than transplantation performed through a nasogastric or nasoduodenal tube which is $81 \%$. The success rate of transplantation through capsules by mouth and colonoscopic administration are equal [8] (Figure 1).

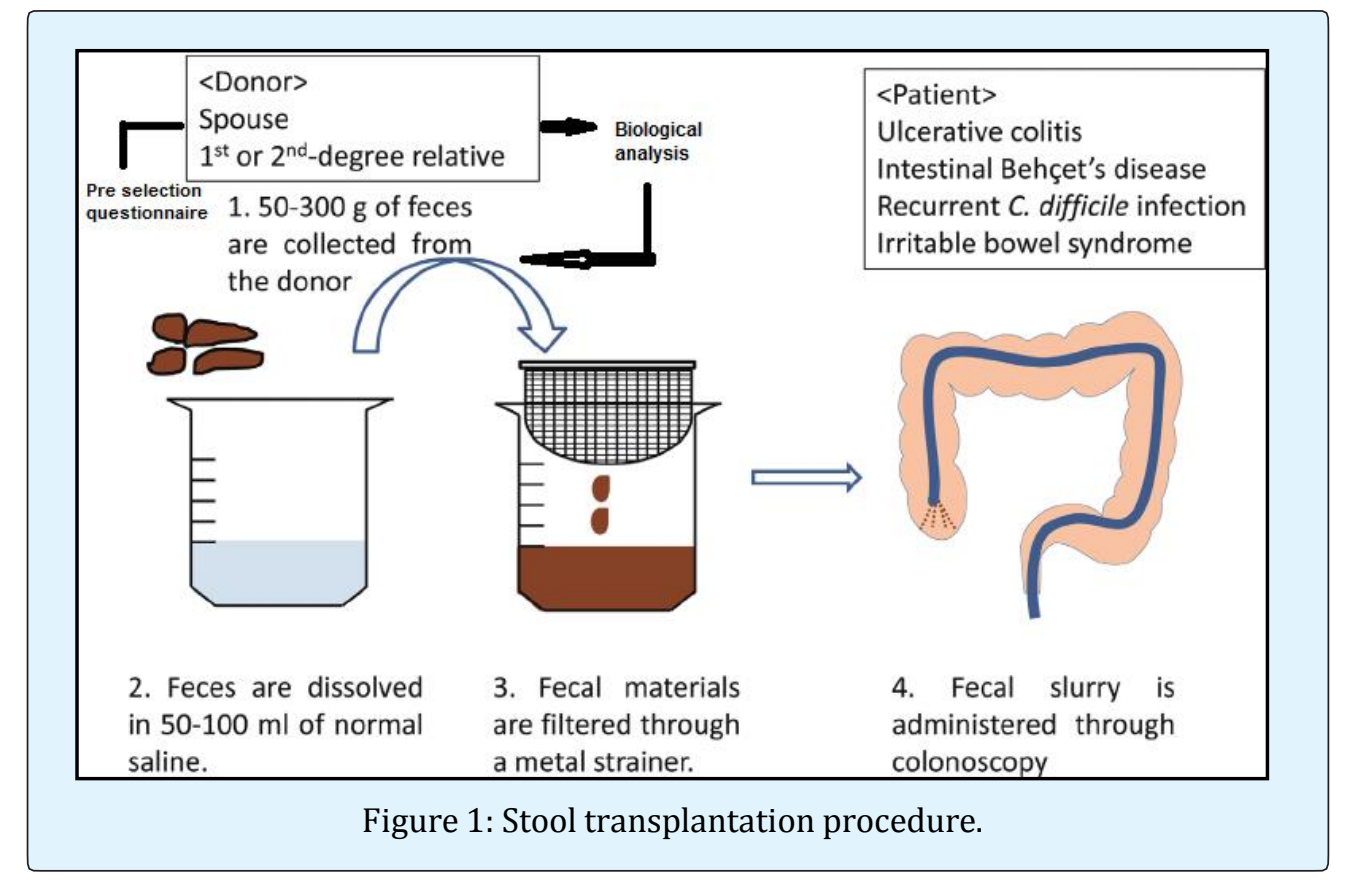

Rajani Chowdary A. A Review on Stool Transplantation. J Inf Dis Trav Med 2018, 2(1): 000106. 


\section{Journal of Infectious Diseases \& Travel Medicine}

\section{Side Effects of Fecal Transplantation}

Some studies have reported the side effects due to stool transplantation which include blood borne infections of bacteria, fever, aggravation of irritable bowel syndrome in people who also had that condition, and mild gastrointestinal distress which generally resolved soon after the procedure including flatulence, diarrhea, irregular bowel movements, abdominal distension, abdominal pain, constipation, cramping, and nausea.

\section{Future Directions}

Now-a-days FMT rate is slowly increasing and the acceptance is also steadily increases, this is due to its consciousness as a natural treatment, and its relatively inexpensive transplantation [9]. Regardless of this perception, there are some fears regarding the infectious potential of the therapy. This gives an insight to some researchers to investigate the use of 'synthetic stool' products with defined bacterial populations to upgrade such issues. Currently many attempts are in underway to find out the role of gut microbiota in the patho-physiology of many other conditions, including necrotizing enterocolitis, liver disease, colorectal cancer, esophageal and gastric adenocarcinoma, and autism [10]. In addition to these, watching the patients often treated with stool transplantation for functional bowel disorders have noted improvement in seemingly unrelated co-morbidities, result a possible role for gut intestinal flora modification in many other conditions. Based on these findings, FMT may be considered a potentially useful therapy for additional conditions in the future.

\section{References}

1. Eiseman B, Silen W, Bascom GS, Kauver AJ (1958) Fecal enema as an adjunct in the treatment of pseudomembranous enterocolitis. Surgery 44(5): 854-859.

2. Turnbaugh PJ, Hamady M, Yatsunenko T, Cantarel BL, Duncan A, et al. (2009) A core gut microbiome in obese and lean twins. Nature 457(7228): 480-484.
3. Dominguez Bello MG, Costello EK, Contreras $\mathrm{M}$, Magris M, Hidalgo G, et al. (2010) Delivery mode shapes the acquisition and structure of the initial microbiota across multiple body habitats in newborns. Proc Natl Acad Sci 107(26): 11971-11975.

4. Gianotti RJ, Moss AC (2017) Fecal Microbiota Transplantation: From Clostridium difficile to Inflammatory Bowel Disease. Gastroenterol hepatol 13(4): 209-213.

5. Rossen NG, Fuentes S, van der Spek MJ, Tijssen JG, Hartman JH, et al. (2015) Findings from a randomized controlled trial of fecal transplantation for patients with ulcerative colitis. Gastro enterology 149(1): 110118.

6. Vaughn BP, Vatanen T, Allegretti JR, Bai A, Xavier RJ, et al. (2016) Increased intestinal microbial diversity following fecal microbiota transplant for active Crohn's disease. Inflamm Bowel Dis 22(9): 21822190.

7. Fischer M, Kao D, Kelly C, Kuchipudi A, Jafri SM, et al. (2016) Fecal microbiota transplantation is safe and efficacious for recurrent or refractory Clostridium difficile infection in patients with inflammatory bowel disease. Inflamm Bowel Dis 22(10): 2402-2409.

8. Kelly CR, de Leon L, Jasutkar N (2012) Fecal microbiota transplantation for relapsing Clostridium difficile infection in 26 patients: methodology and results. J Clin Gastroenterol 46(2): 145-149.

9. Kelly C, Kahn S, Kashyap P, Laine L, Rubin D, et al. (2015) Update on fecal microbiota transplantation 2015: indications, methodologies, mechanisms, and outlook. Gastroenterology 149(1): 223-237.

10. Buie $\mathrm{T}$ (2015) Potential etiologic factors of microbiome disruption in autism. Clin Ther 37(5): 976-983. 